UCRL-JRNL-203630

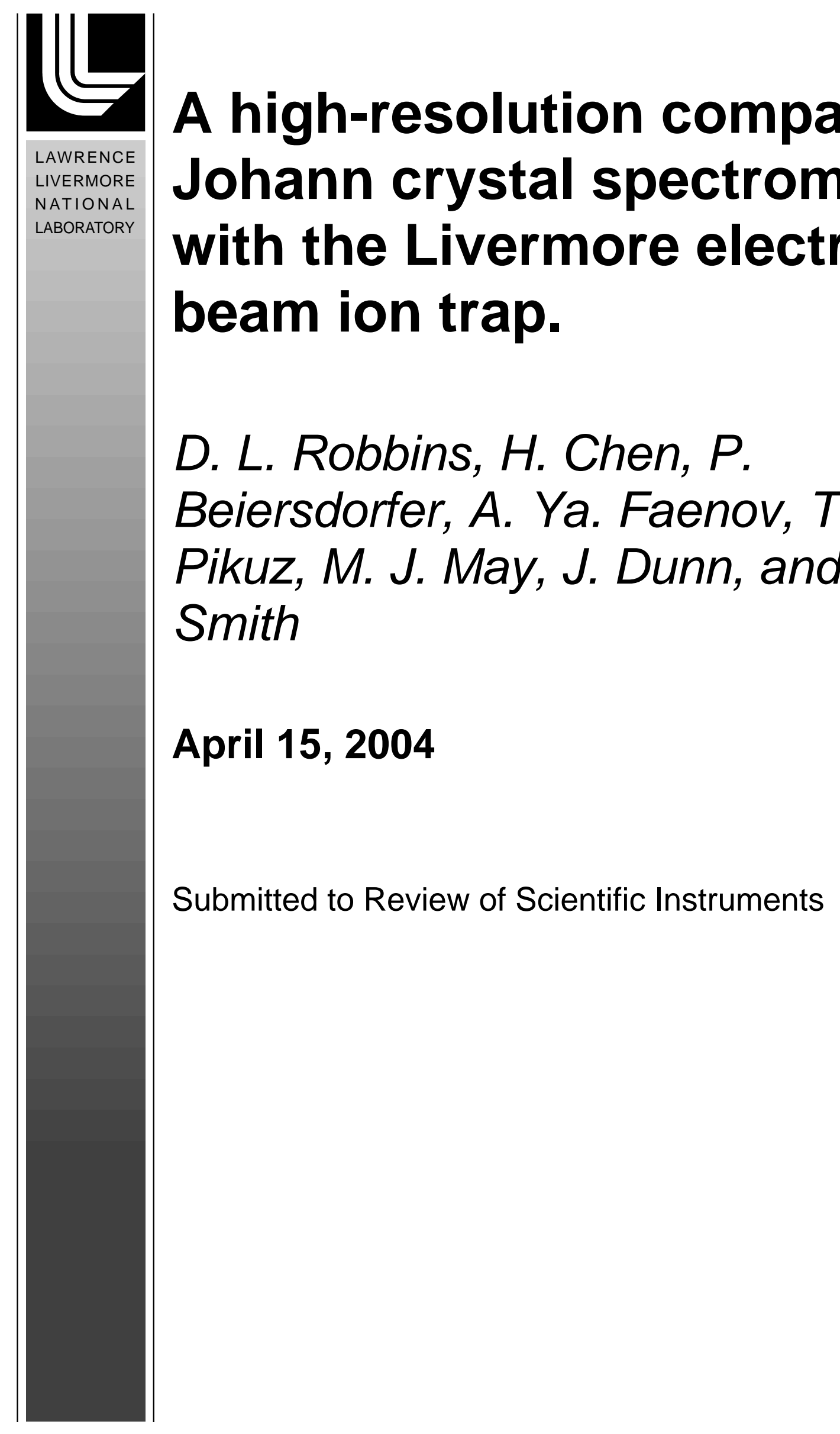


This document was prepared as an account of work sponsored by an agency of the United States Government. Neither the United States Government nor the University of California nor any of their employees, makes any warranty, express or implied, or assumes any legal liability or responsibility for the accuracy, completeness, or usefulness of any information, apparatus, product, or process disclosed, or represents that its use would not infringe privately owned rights. Reference herein to any specific commercial product, process, or service by trade name, trademark, manufacturer, or otherwise, does not necessarily constitute or imply its endorsement, recommendation, or favoring by the United States Government or the University of California. The views and opinions of authors expressed herein do not necessarily state or reflect those of the United States Government or the University of California, and shall not be used for advertising or product endorsement purposes.

This work was performed under the auspices of the U.S. Department of Energy by University of California, Lawrence Livermore National Laboratory under Contract W-7405-Eng-48. 


\title{
A high-resolution compact Johann crystal spectrometer with the Livermore electron beam ion trap.
}

\author{
D. L. Robbins ${ }^{1}$, H. Chen², P. Beiersdorfer ${ }^{2}$, A. Ya. Faenov ${ }^{3}$, T. A. Pikuz ${ }^{3}$, M. J. May², J. \\ Dunn $^{2}$, and A. J. Smith ${ }^{1}$ \\ ${ }^{I}$ Department of Physics, Morehouse College, Atlanta, GA 30314 \\ ${ }^{2}$ Lawrence Livermore National Laboratory, Livermore, CA 94550 \\ ${ }^{3}$ Multicharged Ions Spectra Data Center of VNIIFTRI, Mendeleevo, Moscow region, \\ 141570 Russia
}

\begin{abstract}
A compact high-resolution $(\lambda / \Delta \lambda \approx 10000)$ spherically bent crystal spectrometer in the Johann geometry was recently installed and tested on the Lawrence Livermore National Laboratory SuperEBIT electron beam ion trap. The curvature of the mica (002) crystal grating allows for higher collection efficiency compared to the flat and cylindrically bent crystal spectrometers commonly used on the Livermore electron beam ion traps. The spectrometer's Johann configuration enables orientation of its dispersion plane to be parallel to the electron beam propagation. Used in concert with a crystal spectrometer, whose dispersion plane is perpendicular to the electron beam propagation, the polarization of $\mathrm{x}$-ray emission lines can be measured.
\end{abstract}

\section{INTRODUCTION}

The EBIT and SuperEBIT electron beam ion traps, originally designed at the

Lawrence Livermore National Laboratory, are unique devices specifically design to study highly charge ions [1]. It has been successfully used to infer cross sections, line position, and line intensity ratios for a variety of astrophysically important plasmas [2-5]. Due to excitation by directional electrons in the trap, the x-ray line emission of the trapped ions are generally polarized. Corrections for polarization are needed for accurate interpretation of spectra taken with instruments sensitive to polarization. It should also 
be noted that polarization is a phenomenon that has a potential to be used as a plasma diagnostic tool to infer the presence of electrons beams in both laboratory and Space born plasma sources [6-9].

A compact, spherically curved crystal spectrometer arranged in the Johann geometry equipped with a mica (002) crystal and charged couple device (CCD) was recently installed and tested on one of the Livermore electron beam ion traps. The curvature of the mica crystal allows for greater collection efficiency compared to the flat crystal spectrometers (FCS) commonly used for measurements on an electron beam ion trap $[10,11]$. This higher collection efficiency is a big advantage given the low $\mathrm{x}$-ray production rate of EBIT.

In this brief article we describe the instrument and highlight the advantages of using a compact curved crystal spectrometer in the Johann configuration with the Livermore electron beam ion trap. Very importantly, this instrument reduces the complexity of measuring the polarization of $\mathrm{x}$-ray line emission. We illustrate this capability by showing a polarization measurement of the heliumlike sulfur resonance line $1 \mathrm{~s} 2 \mathrm{p}^{1} \mathrm{P}_{1} \rightarrow 1 \mathrm{~s}^{2}{ }^{1} \mathrm{~S}_{0}$. Moreover, this new instrument can record a full K-shell spectrum in a single setting with high resolution. By contrast, a spherically bent crystal spectrometer had been used earlier on EBIT [12]. This instrument required two settings to record the K-shell spectrum of a heliumlike ion, and therefore has not been used since. 


\section{EXPERIMENTAL SETUP}

Figure 1(a) illustrates the geometry of the compact spherical crystal spectrometer arranged in the conventional Johann configuration [13]. The x-ray source, SuperEBIT, is located outside of the Rowland Circle while the mica crystal grating and CCD are placed on the circle. The Bragg angle of an incident $\mathrm{x}$-ray photon is given by,

$$
\varphi=\sin ^{-1}\left[\frac{2 d}{n \lambda}\right]
$$

where $2 \mathrm{~d}$ denotes the lattice spacing (the mica crystal has a 2d-lattice spacing of 19.942 $\AA$ ), $n$ represents the order of diffraction, and $\lambda$ the wavelength. The distances $a$ and $b$ labeled in Fig 1(a) are the distance between the SuperEBIT source and the crystal and the distance between the crystal and the x-ray image, respectively. In order to obtain optimal spatial resolution the distances a and $\mathrm{b}$ must be equal to the following $[13,14]$,

$$
\begin{gathered}
a=\frac{R \cdot \cos \left[90^{\circ}-\varphi_{o}\right]}{\cos \left[2 \cdot\left(90^{\circ}-\varphi_{o}\right)\right]} \\
b=R \cdot \cos \left[90^{\circ}-\varphi_{o}\right]
\end{gathered}
$$

In equations (2) and (3), R is the radius of curvature of the spherical crystal while $\varphi_{0}$ denotes the incident angle of incoming x-ray photon.

We employ a radius if curvature of $10 \mathrm{~cm}$. As a result, the spherical crystal spectrometer has a compact size of $200 \times 100 \times 100 \mathrm{~mm}^{3}$. It has a wide spectral range of 
$1.2-19.6 \AA$ which can be obtain at one crystal setting by making use of the many different orders of reflection of mica, most of which have high reflectivity [15]. The spectrometer has a resolving power of $\sim 10000$ with a spatial resolution of $\sim 40-80 \mu \mathrm{m}$, which is enough to resolve lines in typical heliumlike ions' $\mathrm{K} \alpha$ transitions. In order to make use of the narrow line focus of the compact spectrometer it is necessary to use a detector with a spatial resolution less than the focal width. In this case, we used a $1024 \times$ 1024 pixel x-ray CCD camera operating in vacuo. Each pixel has a dimension of $24 \mu \mathrm{m}$ $\times 24 \mu \mathrm{m}$. The CCD is water cooled and reaches $-40{ }^{\circ} \mathrm{C}$ with the help of a Peltier cooler [16].

Figure 1(b) shows a diagram of the spherical crystal spectrometer connected with the Livermore SuperEBIT apparatus. Also shown in Fig 1(b), is a flat crystal spectrometer that records complementary data. The FCS employs a dispersion plane perpendicular to the electron beam propagation while the compact spherical crystal spectrometer dispersion is in the direction of the electron beam propagation (see Fig 1(b) for illustration). These two orientations are ideal for polarization measurements using the "two-crystal technique" [17]. 


\section{MEASUREMENT AND ANALYSIS}

Shown in Fig 2 are two spectra of the heliumlike $\mathrm{S}^{14+} \mathrm{K} \alpha$ transitions taken at an electron beam energy of $3 \mathrm{keV}$. The top spectrum was recorded by the compact spherical crystal spectrometer, while the bottom spectrum was recorded by the FCS describe in the previous section. For the spherical spectrometer, the K $\alpha$ transitions where observed in third order $(n=3)$ at a Bragg angle of $\theta_{B}=49 \cdot 6^{\circ}$. The FCS was equipped with a pentaerythritol crystal with a 2d-lattice spacing of $8.742 \AA$, resulting in a Bragg angle of $\theta_{\mathrm{B}}=35.2^{\circ}$ for the $\mathrm{K} \alpha$ transitions in heliumlike sulfur. The intensities observed by the crystal spectrometers can be expressed as,

$$
I^{o b s}=R_{\|} I_{\|}+R_{\perp} I_{\perp}
$$

where $R_{\|}$, and $R_{\perp}$ represent the integrated crystal reflectivities for x-ray emission polarized parallel and perpendicular to the plane of dispersion, respectively. $I_{\|}$and $I_{\perp}$ denote the intensity of the emitted radiation with an electric field vector parallel and perpendicular to the electron beam direction, respectively. The polarization of emission lines observed at an angle of $\vartheta=90^{\circ}$ from the electron beam are defined as,

$$
P=\frac{I_{\|}-I_{\perp}}{I_{\|}+I_{\perp}}
$$

The two crystal spectrometers act as polarimeters. The FCS is oriented in a geometry that preferably reflects $I_{\|}$more efficiently than $I_{\perp}$. The spherical crystal spectrometer absorbs most of $I_{\|}$while reflecting $I_{\perp}$. 
After fitting the two spectra shown in Fig 2, the polarization of the resonance line, $1 \mathrm{~s} 2 \mathrm{p}^{1} \mathrm{P}_{1} \rightarrow 1 \mathrm{~s}^{2}{ }^{1} \mathrm{~S}_{0}$, is measured at $P_{w}=+0.55_{-0.15}^{+0.15}$ which compares very well with the

predictions of distorted-wave, $P_{w}^{\text {Theroy }}{ }_{-}^{D W}=+0.61$.

\section{ACKNOWLEDGEMENT}

We gratefully acknowledge the support by the LLNL Research Collaborations Program for Historical Black Colleges and Universities. This work was performed under the auspices of the U. S. Department of Energy by Lawrence Livermore National Laboratory under Contract No. W-7405-ENG-48 and by Morehouse College under Contract No. DE-FG02-98ER14877. We also acknowledge the NATO Collaborative Linkage Grant PST.CLG.97889. 


\section{FIGURE CAPTIONS}

FIG. 1. (a) Geometry of the Compact Johann Spherical Crystal Spectrometer. (b) Electron beam ion trap x-ray polarization measurement set-up (modified "two-crystal technique"). FCS preferentially reflects $I_{\|}$, while the spherically bent crystal spectrometer reflects $I_{\perp}$. The CCD detector for the spherically bent crystal spectrometer is located below the crystal housing and is not shown.

FIG. 2. Spectra obtained with (a) the compact spherically bent crystal spectrometer (b) and the flat crystal spectrometer. The electron beam energy $\sim 3.0 \mathrm{keV}$. 


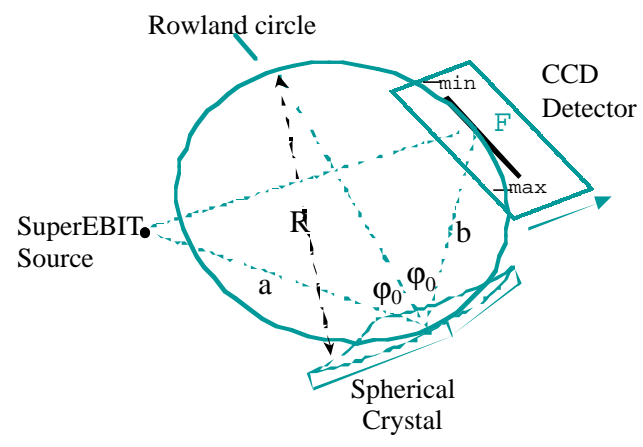

FIG. 1(a)

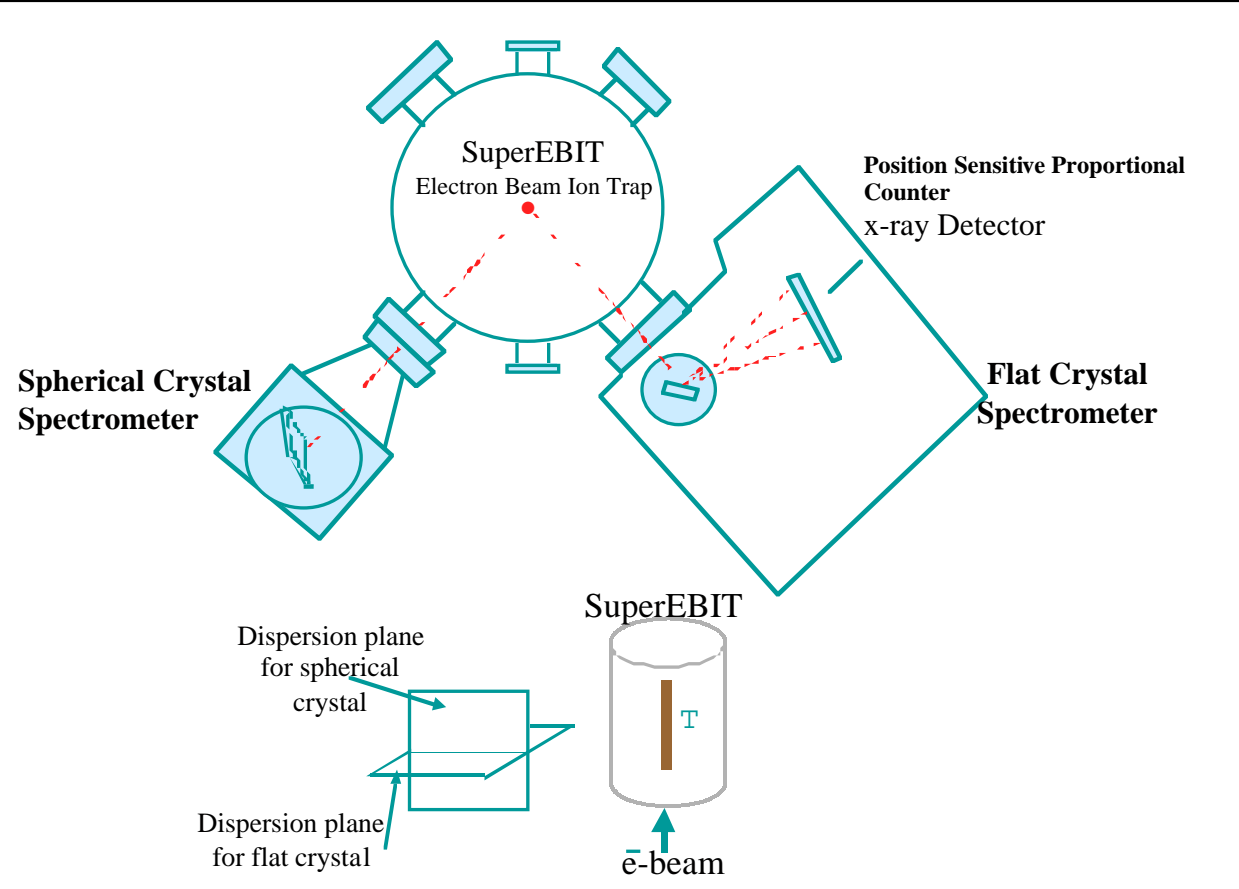

FIG. 1(b) 


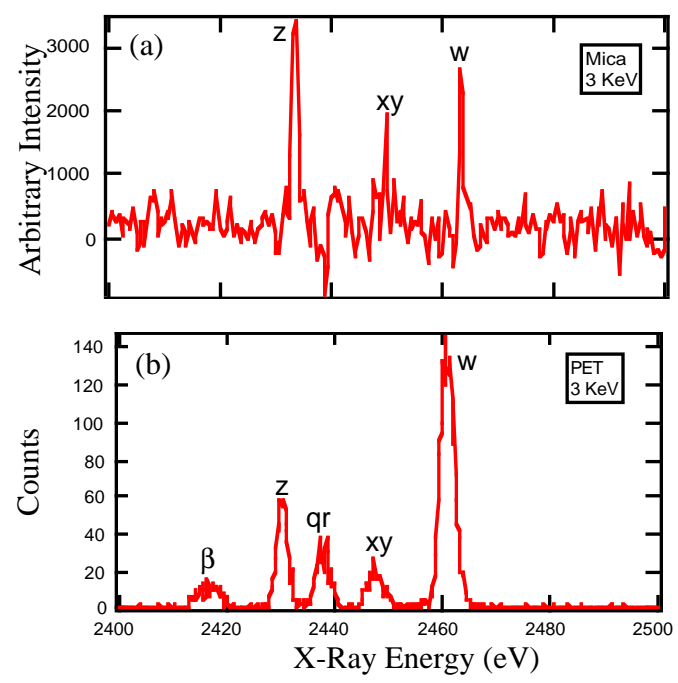

FIG. 2 


\section{REFERENCES}

${ }^{1}$ M.A. Levine, R.E. Marrs, J.N. Bardsley, P. Beiersdorfer, C.L. Bennett, M.H. Chen, T. Cowan, D. Dietrich, J.R. Henderson, D.A. Kanpp, A. Osterheld, B.M. Penetrente, M.B. Schneider, and J.H. Scofield, Nucl. Instrum. Methods B43, 431 (1989).

${ }^{2}$ G. 'V. Brown, P. Beiersdorfer, D.A. Liedahl, and K. Widmann, ApJ, 502, 1015 (1998).

${ }^{3}$ M.F. Gu, S.M. Kahn, D.W. Savin, E. Bahar, P. Beiersdorfer, G.V. Brown, D.A. Liedahl, and K.J. Reed, ApJ, 563, 462 (2001).

${ }^{4}$ M.F. Gu, D.W. Savin, and P. Beiersdorfer, J. Phys. B, 32, 5371 (1999).

${ }^{5}$ H. Chen, P. Beiersdorfer, J.H. Scofield, K.C. Gendreau, K.R. Boyce, G.V. Brown, R.L. Kelley, F.S.

Porter, C.K. Stahle, A.E. Szymkowiak, and S.M. Kahn, ApJ 567, L169 (2002).

${ }^{6}$ M.K. Inal, J. Dubau, J. Phys. B: At. Mol. Phys. 20, 4221 (1987).

${ }^{7}$ E. Haug, Sol. Phys. 71, (1981).

${ }^{8}$ Proceedings of $3^{\text {rd }}$ US-Japan Plasma Polarization Spectroscopy Workshop, Livermore, CA, June 1821, 2001, University of California LLNL Rp. UCRL-ID-146907, edited by P. Beiersdorfer and T. Fujimoto. ${ }^{9}$ R. Beier, C. Bachmann, and R. Burhenn, J. Phys. D 14, 643 (1981).

${ }^{10}$ G.V. Brown, P. Beiersdorfer, and K. Widmann, Rev. Sci. Instrum. 70, 280 (1999).

${ }^{11}$ P. Beiersdorfer, J. R. Crespo López-Urrutia, E. Förster, J. Mahiri, and K. Widmann Rev. Sci. Instrum. 68, 1077 (1997).

${ }^{12}$ J.R. Henderson, P. Beiersdorfer, C.L. Bennett, S. Chantrenne, D.A. Knapp, R.E. Marrs, M.B. Schneider, K.L. Wong, G.A. Doschek, J.F. Seely, C.M. Brown, R.E. LaVilla, J. Dubau, and M.A. Levine, Phys. Rev. Lett. 65, 705 (1990).

${ }^{13}$ H.H. Johann, Z. Phys. 69, 185 (1931).

${ }^{14}$ P. Beiersdorfer, S. von Goeler, M. Bitter, K.W. Hill, R.A. Hulse, and R.S. Walling, Rev. Sci. Instrum. 60, 895 (1989).

${ }^{15}$ S. G. Lee, J. G. Bak, Y. S. Jung, M. Bitter, K. W. Hill, G. Hölzer, O. Wehrhan, and E. Förster Rev. Sci. Instrum. 74, 5046 (2003).

${ }^{16}$ J. Dunn, B.K.F. Young, A.D. Conder, and R.E. Stewart, Proceedings of SPIE 2654, 119 (1996).

${ }^{17}$ P. Beiersdorfer, D.A. Vogel, K.J. Reed, V. Decaux, J.H. Scofield, K. Widmann, G. Hölzer, E. Förster, O. Wehrhan, D.W. Savin, and L. Schweikhard, Phys. Rev. A 53, 3974 (1996). 